\title{
Accuracy Improvement of Compressed Video using (IWD) Intelligent Water Droplet
}

\author{
Akriti Kashyap \\ M.TECH \\ Research Scholar \\ Department of Electronics and Communication \\ RIEIT, Ropar
}

\author{
Sukhwinder Kaur \\ Associate Professor \\ Department of Electronics and Communication \\ RIEIT, Ropar
}

\begin{abstract}
In this video compression is done, video is converted into number of frame and over these frame compression algorithm is implemented. In number of frame IWD(intelligent water droplet) algorithm is implemented. On the basis of its implementation,matrices will be generated and by these matrices compressed video will be formed.
\end{abstract}

\section{Keywords}

Video compression, Markov, IWD, PSNR, MSE , EPI\& SSIM.

\section{INTRODUCTION}

With advancement of time, as technology developing day by day quality of multimedia product also increasing .As quality of multimedia product also increasing, it take more memory space for storage and hence will use more data when shared from one network to other network. So, to overcome this problem data compression techniques are introduced. From image to video we have different compression [1] technique [13]. Our main aim is to reduce its size without changing its quality.

As nowadays high definition cameras is used, by increasing quality of image number of bits involved also increases. As if we calculate number of bits in HD image it will be $512 * 512 * 8=2097152$ bits [10]. In case of video it will be more than 2097152 bits. So compression is must for video when it has to be shared over network.

In this paper, intelligent water droplet algorithm (IWD) [5] is used for video compression [15] . It will enhance compression ratio, PSNR \& reduce MSE.

\section{METHODOLOGY}

In proposed model, the intelligent water droplet (IWD) is used to optimize compression. In this paper firstly video [3] is biparted [14] video, then on each frame IWD [4] is implemented, afterward video [17] is to be reconstruct from generated matrix. The quality of recovered video will be checked by calculating compression ratio, PSNR, MSE.

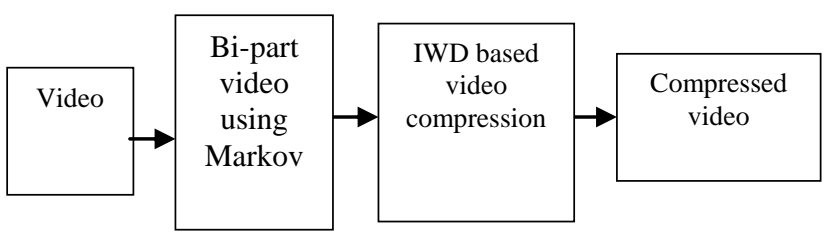

Figure 1: Flow chart of our proposed algorithm

\subsection{ALGORITHM}

The algorithm design is shown in Figure 3.2, which involves:

Step 1: Video Acquisition.

Step 2: Bi-part the whole video through markovs.

Step 3: IWD [16] Parameter initialization (Coefficients of video)

- $\quad$ Static Parameter initialization:

The number of water drops $N_{I W D}$ is set to a positive integer value, which is usually set to the number of values $N_{c}$ of the graph.

For velocity updating, the parameters are $a_{v}=1, \mathrm{bv}=.01$ and $c_{v}=1$.

- Dynamic Parameter initialization: Soil \& velocity of IWDs

Every IWD has a visited node list $\mathrm{V}_{\mathrm{c}}$ (IWD), which is initially empty: $V_{c}(I W D)=\{\}$. Each IWD's velocity is set to Init Vel. All IWDs are set to have zero amount of soil.

Step 4: Calculate mean values of bi-part videos which is equal soil content of the bi-part video.

Step 5: On the basis of water drop, Depart matrices by adding water drops one by one. 


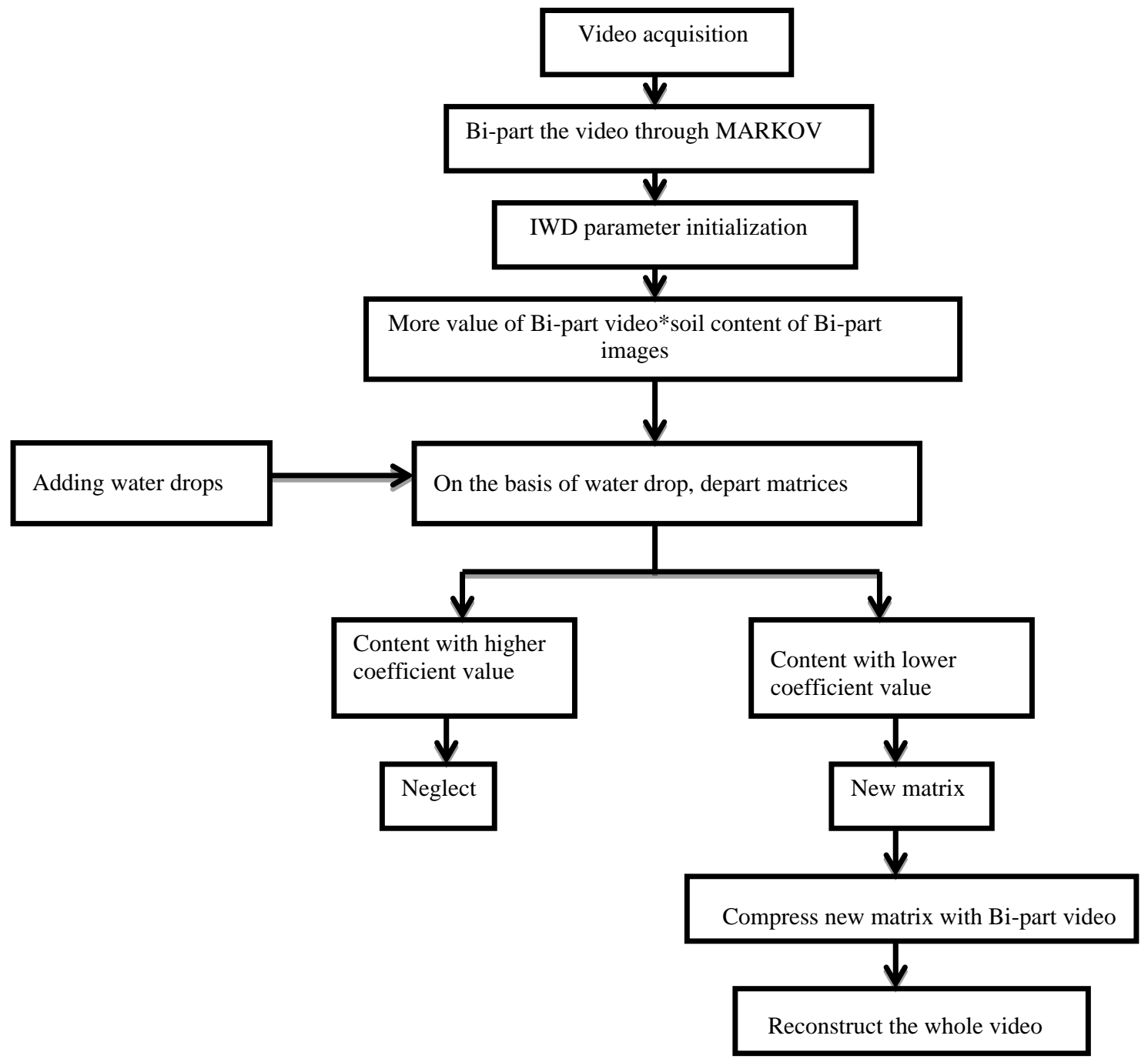

Figure 2: Flow chart of our proposed algorithm.

$$
v e l^{I W D}(t+1)=\operatorname{vel}^{I W D}(t)+\frac{a_{v}}{b_{v}+c_{v} \cdot \operatorname{soil}^{2}(i, j)}
$$

Wherever $l^{I W D}(t+1)$ is the updated velocity of the IWD.

Step 7: Solution construction by IWDs along with water drop velocity updating.

- Neglect the content with higher soil values.

- Continue with lower soil values.

Step 8: Generate a new matrix \& compare new matrix with bipart videos.

Step 9: Reconstruct the whole video.

\section{Video Acquisition}

In this proposed work, we used a sample of 10 videos numbered as 0 to 9 . We collect all samples with the help of

help of internet. All videos are gray scaled and we converted all samples to same size .

Video Bi-parts (frames)

Bi-parts the original video using markovs. Fig 4 shows the original video and Bi-parts video. This bi-part video is used for further process of this proposed system.
The original video \& compressed video using our proposed IWD [2] based Video compression technique. The compressed video is less distorted using intelligent water drop system based video compression [7] scheme.

\section{EXPERIMENTAL RESULTS}

\subsection{Video Frames}

As all knows it is not possible to work on video directly, so bi-partition is required. Video is to be break in different number of frame and apply IWD on them and after compressing video, calculated frame will be recombine to get compressed video. As in this it will have 69 frames

\subsection{Implementation}

To implement IWD algorithm, it has two parameter static and dynamic parameter. Static parameter include number of water drops, velocity updating parameter and dynamic parameter include number of visited node. 


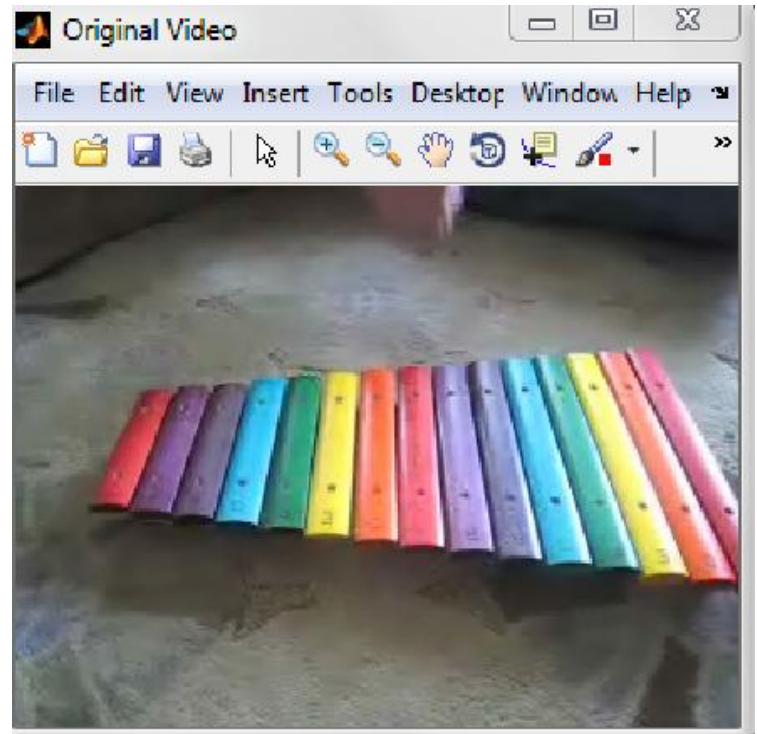

Figure 3: Original Video.

Initially, static parameter: number of water drops is set to positive integer value and velocity updating parameters are set to unity. Dynamic parameter: number of visited node list is empty and velocity of IWD [11] is initially zero. All drop have zero amount of soil.

Mean value for bi-part video will be calculated. We will form number of matrices on the basis of water drops, for every intelligent water droplet different velocity will be updated. After calculating number of value, it will continue with lower soil content value only and neglect all higher content value. By using lower soil content value it will generate new matrices. Hence reconstruct the whole video

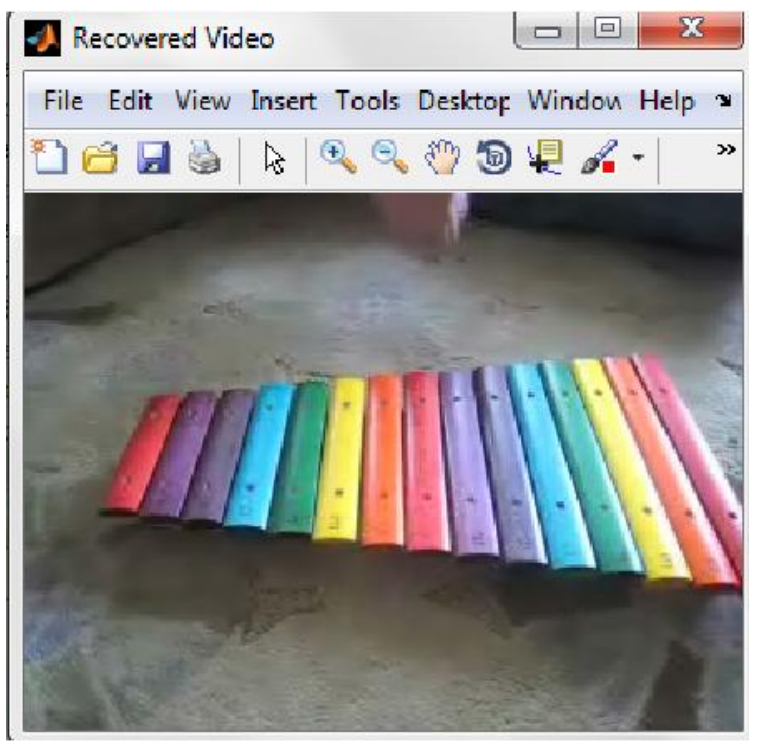

Figure 4: recovered video

\subsection{Result}

After reconstructing video from calculated matrices output will be compressed or recovered video. By calculating different parameter PSNR, MSE, EPI \& SSIM for recovered video quality of video will be checked. Accuracy of IWD is being calculated and achieved up to $95-100 \%$

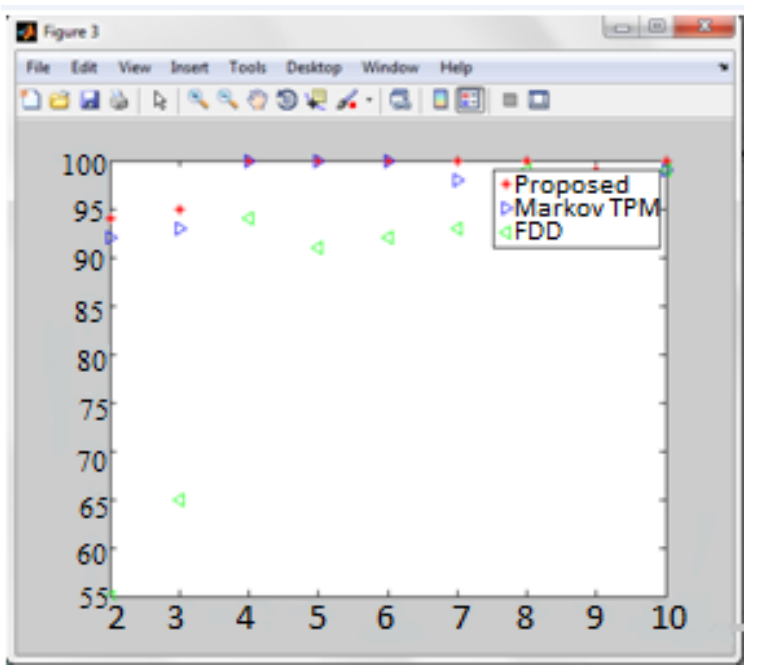

Figure 5: Accuracy graph

After implementing IWD, compressed video is achieved. It can be observed that original and compressed video is almost same. Hence compression without losing its actual quality is achieved.

In figure 5, accuracy of IWD is plotted, Markov TPM [12] and FDD. Accuracy of FDD [6] [8] [9] is lowest while IWD \& Markov have almost same accuracy but when qs 1 is 2,37 \& 8 accuracy of Markov [18] has been dropped while IWD has maintained its accuracy.

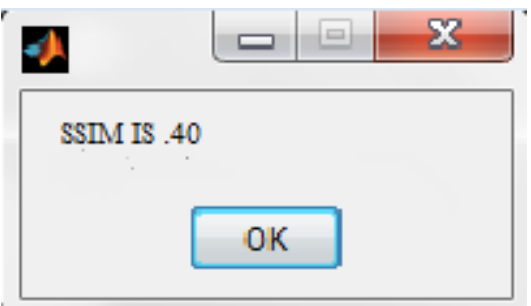

Figure 6: Calculated SSIM

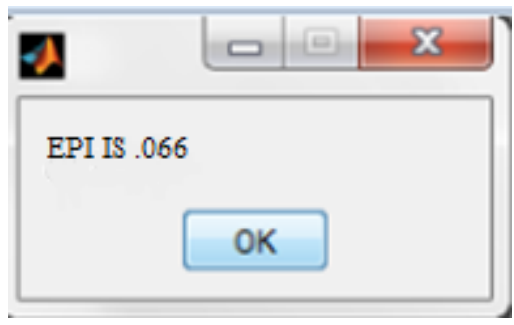

Figure 7: Calculate EPI

In proposed work minimum MSE is calculated and maximum PSNR. In table 1 calculated parameter and its calculated value are written.

Table 1. Calculated Errors

\begin{tabular}{|c|c|c|}
\hline Sno. & Parameters & $\begin{array}{c}\text { Calculated } \\
\text { Values }\end{array}$ \\
\hline 1 & $\begin{array}{c}\text { COMPRESSION } \\
\text { RATIO }\end{array}$ & 1 \\
\hline 2 & MSE & 0.07 \\
\hline
\end{tabular}




\begin{tabular}{|c|c|c|}
3 & $\begin{array}{c}\text { RUNNING } \\
\text { TIME }\end{array}$ & 0.28 \\
\hline 4 & SSIM & 0.4 \\
\hline 5 & EPI & 0.066 \\
\hline 6 & PSNR & 89 \\
\hline
\end{tabular}

\section{CONCLUSIONAND FUTURE SCOPE}

The main motive of the proposed work is to improve the accuracy of recovered video. it has achieved higher accuracy than previous work. In table 2, comparison of accuracy of previous and proposed is shown work. Proposed work has achieved better result

Table 2: Comparison of previous and present work

\begin{tabular}{|c|c|c|}
\hline $\begin{array}{c}\text { QS1(DOUBLE } \\
\text { COMPRESSED } \\
\text { COEFFICIENT) }\end{array}$ & $\begin{array}{c}\text { MARKOV(PREVIOUS } \\
\text { WORK) }\end{array}$ & $\begin{array}{c}\text { IWD(PROPOSED } \\
\text { WORK) }\end{array}$ \\
\hline 2 & 92.3 & 94.5 \\
\hline 3 & 93.5 & 95.1 \\
\hline 4 & 100 & 100 \\
\hline 5 & 100 & 100 \\
\hline 6 & 100 & 100 \\
\hline 7 & 98.3 & 100 \\
\hline 8 & 94.6 & 100 \\
\hline 9 & 98.9 & 99.5 \\
\hline 10 & 99.1 & 99.8 \\
\hline & & \\
\hline
\end{tabular}

For future, it has wide area of research; work on various parameter of video like motion estimation, recognition which will also contribute for compression with better quality.

\section{ACKNOWLEDGMENT}

My sincere thank and gratitude to my esteemed and honorable supervisor Sukhwinder Kaur Associate Professor. She has not only been very kind to me but has always stood as an epitome of knowledge and encouragement. My special thanks to Dr. Ramesh Chand Kashyap, Head of the Department of Electronics and communication Engineering, for his support and providing all those facilities required for the completion of this thesis. Also thankful to the faculty and staff members of Electronic and Communication Engineering Department for helping me out in one way or the other

\section{REFERENCES}

[1] R.C.Gonzalez and R.E.Woods, 2002 on Error free compression in digital image processing.

[2] H.S.Hosseini 2007 on Problem solving by intelligent water drops.

[3] C.-H. Chen, Y.-Q. Shi, and W. Su 2008 on A machine learning based scheme for double JPEG compression detection

[4] Hamed shah-hosseini 2008 on Intelligent water drops algorithm a new optimization method for solving the multiple knapsack problem

[5] H.S.Hosseini 2009 The intelligent water drops algorithm: a nature-inspired swarm-based optimization algorithm

[6] W. Chen and Y.-Q. Shi 2009 on Detection of double MPEG compression based on first digit statistics

[7] Pooja Nagpal, Seema bhagla 2011 on Video compression by memetic algorithm

[8] D.-D. Liao, R. Yang, H.-M. Liu, J. Li, and J.-W. Huang 2011 on Double H.264/AVC compression detection using quantized non zero AC coefficient.

[9] T.-F. Sun, W.Wang, and X.-H. Jiang 2012 on Exposing video forgeries by detecting MPEG double compression

[10] Mr. M.B. Bhammar K.A. Mehta.2013 on Performance improvement of SPIHT algorithm using hybrid image compression technique.

[11] Priti Aggarwal, Harish Kundra 2013 on Image restoration using intelligent water drops.

[12] Xinghao Jiang, Wan Wang, Tanfeng Sun,Atal, 2013 on Detection of double compression in MPEG-4 video based on markov statistics.

[13] M.Atheeshwari, K.Mahesh 2014 on Video compression technique - A comprehensive survey.

[14] Muhammad Aakif Shaikh, Prof. Sagar S. Badnerkar 2014 on Video compression algorithm using motion compensation technique

[15] T.Bernatin , G.Sundari 2014 on Video compression based on hybrid transform and quantization with Huffman coding for video codec

[16] Manmeen Kaur Gaganpreet Kaur Dheerendra Singh 2014 on Optimization using Bio-Inspired algorithm: Intelligent water drop algorithm.

[17] Priya Rani, Alka Kumari 2015 on A survey on efficient memory in video codec using hybrid algorithm

[18] S.Bhuvaneshwari, Dr.T.Gunasekaran 2015 on A review on effective compression in MPEG-4 videos using Markov statistics 